\title{
Latest three-flavor neutrino oscillation results from NOvA
}

\section{Liudmila Kolupaeva*, for the NOvA collaboration}

The Joint Institute for Nuclear Research

E-mail: ldkolupaeva@yandex.ru

The NOvA experiment is a long-baseline neutrino oscillation experiment that uses the upgraded NuMI beam from Fermilab to detect both electron neutrino appearance and muon neutrino disappearance. NOvA employs two functionally identical detectors: a Near Detector, located at Fermilab, and a Far Detector, located at Ash River, Minnesota over an 810 km baseline. NOvA's primary physics goals include precision measurements of neutrino oscillation parameters, such as $\theta_{23}$ and the atmospheric mass-squared splitting, along with probes of the mass hierarchy and the CP violating phase. This document is devoted to the latest NOvA measurements [1] of the neutrino oscillation parameters using neutrino and antineutrino disappearance and appearance.

European Physical Society Conference on High Energy Physics - EPS-HEP2019 -

10-17 July, 2019

Ghent, Belgium

${ }^{*}$ Speaker. 


\section{Introduction}

NOvA [2] is a long-baseline accelerator neutrino experiment devoted to studying the properties of neutrino oscillations. It consists of two functionally identical tracking calorimeter detectors. The NuMI facility [3] at FNAL (USA) creates a muon (anti-)neutrino beam which travels $810 \mathrm{~km}$ through the Earth's crust to the Far Detector, which is placed at Ash River (Minnesota, USA).

The proton NuMI beam with $120 \mathrm{GeV}$ energy is delivered to carbon target. This interaction produces mesons which decay into leptons and neutrinos. The resulting beam composition in 1 - 5 $\mathrm{GeV}$ region is as follows: $95 \% v_{\mu}, 4 \% \bar{v}_{\mu}, 1 \% v_{e}\left(93 \% \bar{v}_{\mu}, 6 \% v_{\mu}, 1 \% v_{e}\right.$ in the case of antineutrino beam). The type of the neutrino beam (whether it consists of neutrinos or antineutrinos) depends on the polarity of the magnetic horns which are used for focusing mesons with a specific charge.

NOvA's design power is $700 \mathrm{~kW}$, which translates to $6 \times 10^{20}$ protons delivered to the target (POT) per year. Currently NOvA has analyzed $8.85 \times 10^{20}$ POT with neutrino beam and $12.33 \times 10^{20}$ POT with antineutrino beam.

The experiment uses two detectors which are placed $14.6 \mathrm{mrad}$ off the beam axis. This scheme helps to obtain narrow energy peak close to $2 \mathrm{GeV}$. The Near Detector (ND) has dimension $4.2 \mathrm{~m}$ $\times 4.2 \mathrm{~m} \times 16 \mathrm{~m}$ and $300 \mathrm{t}$ mass. It is placed about $1 \mathrm{~km}$ after the target and used for measuring the neutrino interaction event rates before oscillations. The Far Detector (FD) is used for measuring the neutrino event rate after oscillations at a distance $810 \mathrm{~km}$. It has dimensions of $15.6 \mathrm{~m} \times 15.6 \mathrm{~m}$ $\times 60 \mathrm{~m}$ and a $14 \mathrm{kt}$ mass.

Both detectors consist of PVC cells filled with a liquid mineral oil based scintillator. Each cell contains a wavelength-shifting fiber loop which is connected with 32-pixel avalanche photodiodes used for readout electronics. Due to the small size of the cells $(4.2 \mathrm{~cm} \times 6 \mathrm{~cm})$ the detector segmentation is very good. Cells are collected into planes with different orientations allowing $3 \mathrm{~d}$ reconstruction.

The NOvA detectors were designed to observe electron and muon (anti-)neutrinos. In order to identify and classify events a Convolutional Visual Network [4] is used.

The resulting neutrino energy spectra at the FD are sensitive to certain oscillation parameters: $\Delta m_{32}^{2}, \theta_{23}, \delta_{C P}$ and the neutrino mass hierarchy. This document will describe NOvA's result [1] produced with data collected between July 2013 and February 2019 with neutrino and antineutrino beams.

\section{Near Detector and Far Detector data}

Similar detector construction allows for the application of the so-called extrapolation procedure: a data-driven technique for producing predictions in the FD. The high statistics of the ND data are used for constraining the simulations. Corrected simulated prediction is extrapolated to the FD and is used for the data fit. This method helps to reduce the size of cross-section and flux systematic uncertainties. A full list of systematic uncertainties for the measured parameters is shown in Table 1.

NOvA's three-flavor oscillation analysis consists of two main interaction channels: electron neutrino appearance and muon neutrino disappearance. 


\begin{tabular}{cccc}
\hline Source & $\sin ^{2} \theta_{23}\left(\times 10^{-3}\right)$ & $\Delta m_{32}^{2}\left(\times 10^{-5} \mathrm{eV}^{2}\right)$ & $\delta_{C P}(\pi)$ \\
\hline \hline Calibration & $+5.4 /-9.2$ & $+2.2 /-2.6$ & $+0.03 /-0.03$ \\
Neutron model & $+6.0 /-13.0$ & $+0.5 /-1.3$ & $+0.01 /-0.00$ \\
Cross-sections & $+4.1 /-7.7$ & $+1.0 /-1.1$ & $+0.06 /-0.07$ \\
$E_{\mu}$ scale & $+2.3 /-3.0$ & $+1.0 /-1.1$ & $+0.00 /-0.00$ \\
Detector model & $+1.9 /-3.2$ & $+0.4 /-0.5$ & $+0.05 /-0.05$ \\
Normalizations & $+1.3 /-2.7$ & $+0.1 /-0.2$ & $+0.02 /-0.03$ \\
ND/FD differences & $+1.0 /-4.0$ & $+0.2 /-0.2$ & $+0.06 /-0.07$ \\
Beam flux & $+0.4 /-0.8$ & $+0.1 /-0.1$ & $+0.00 /-0.00$ \\
\hline \hline Systematic uncertainty & $+9.7 /-20$ & $+2.6 /-3.2$ & $+0.11 /-0.12$ \\
\hline
\end{tabular}

Table 1: Systematic uncertainties for the joint $v_{e}+v_{\mu}$ analysis with neutrino and antineutrino beam.

In the case of muon neutrino disappearance analysis, we split the ND reconstructed energy spectrum into 4 quartiles based on the reconstructed hadronic energy fraction to the full reconstructed neutrino energy $\left(E_{\text {had frac }}=E_{\text {had }} / E_{v}\right)$. Quartile no.1 has the best energy resolution $(\sim 6 \%)$ and the lowest hadronic energy fraction. Whereas quartile no. 4 has the worst energy resolution $(\sim 12 \%)$ and the highest hadronic energy fraction. The resulting measured spectra of ND $v_{\mu}$ Charged Current (CC) candidates are shown in the Figures 1 - 2.
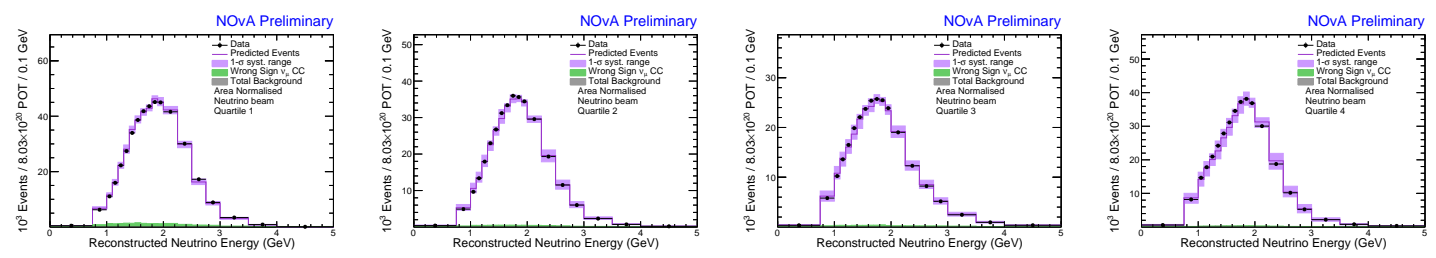

Figure 1: Measured reconstructed energy spectra of ND $v_{\mu}$ CC events (dots) compared to the ND simulation (violet line). Good agreement between simulation and data. High statistics allow to constrain the simulation.
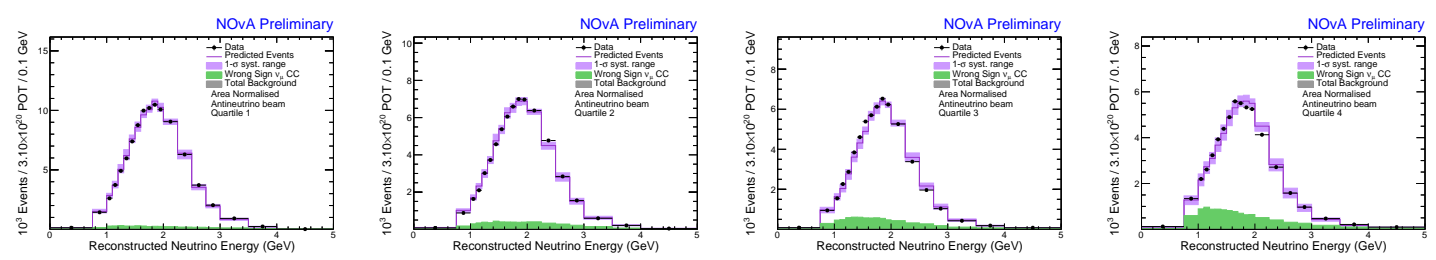

Figure 2: Measured reconstructed energy spectra of ND $\bar{v}_{\mu}$ CC events (dots) compared to the ND simulation (violet line). Good agreement between simulation and data. High statistics allow to constrain the simulation.

Each quartile is separately extrapolated to the far detector. ND selected $v_{\mu}\left(\bar{v}_{\mu}\right)$ events are used in the data-driven prediction of both $v_{\mu}\left(\bar{v}_{\mu}\right)$ and $v_{e}\left(\bar{v}_{e}\right)$ signal events in the FD. Similarly the ND selected $v_{e}\left(\bar{v}_{e}\right)$ CC events are a source of background in the FD. Measured spectra of these events are shown in Figure 3. We split these data into two regions of high and low $v_{e}\left(\bar{v}_{e}\right)$ purity based on their PID score.

In the FD NOvA observed $113 v_{\mu} \mathrm{CC}\left(102 \bar{v}_{\mu} \mathrm{CC}\right)$ candidates with a background expectation $4.2_{-0.6}^{+0.8}$ selected events with neutrino beam and $2.2_{-0.4}^{+0.4}$ events with antineutrino beam. Final 

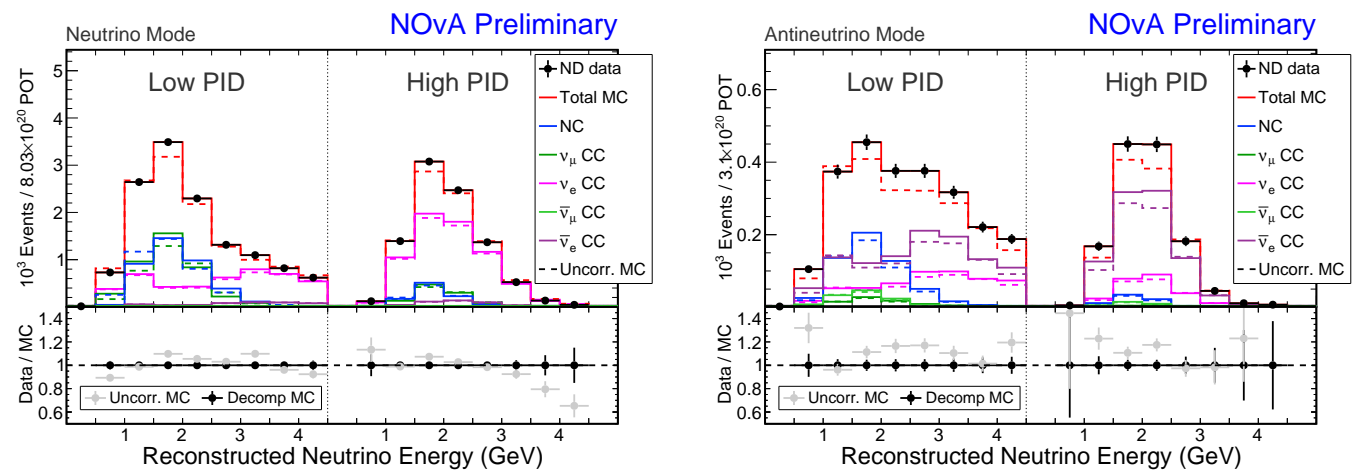

Figure 3: Measured reconstructed energy spectra of ND $v_{e} \mathrm{CC}$ (left) and $\bar{v}_{e} \mathrm{CC}$ (right) events (dots) compared to the ND simulation (color lines). Data-driven techniques are used to correct predictions in order to match the data. Uncorrected simulation before the procedure is shown by dashed lines. Each category of background is extrapolated separately to the FD.

$v_{\mu}$ and $\bar{v}_{\mu}$ spectra are shown in the Figure 4. There are $58 v_{e} \mathrm{CC}\left(27 \bar{v}_{e} \mathrm{CC}\right)$ candidate events with an expected background of $15.0_{-0.9}^{+0.8}$ and $10.3_{-0.5}^{+0.6}$ events for neutrino and antineutrino beam, respectively. Resulting spectra are shown in Figure 5. This observation provides $4.4 \sigma$ evidence of $\bar{v}_{e}$ appearance in an initially predominantly $\bar{v}_{\mu}$ beam.
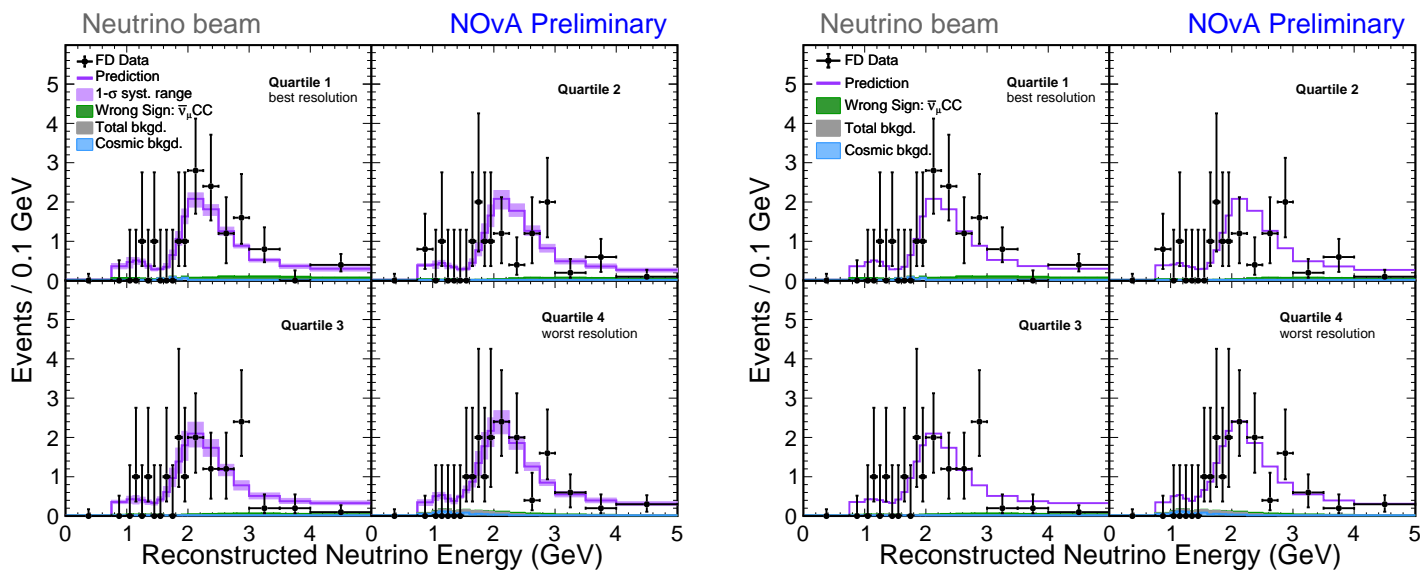

Figure 4: Measured reconstructed energy spectra of FD $v_{\mu} \mathrm{CC}$ (left) and $\bar{v}_{\mu} \mathrm{CC}$ (right) events (dots) split into four quartiles. Color lines show the FD prediction at the best fit value after the extrapolation procedure.

\section{Analysis results}

In order to extract the oscillation parameters, a joint fit of the spectra in Figures 4 - 5 was performed. Solar oscillation parameters $\theta_{12}$ and $\Delta m_{12}^{2}$ were fixed to the PDG values [5] while $\theta_{23}$, $\Delta m_{32}^{2}, \delta_{C P}$ and neutrino mass hierarchy were varied. The value of $\theta_{13}$ was constrained by reactor experiment measurements. NOvA's analysis is frequentist [6] with profiled systematics and penalty terms.

NOvA's best fit is $\Delta m_{32}^{2}=2.48_{-0.06}^{+0.11} \times 10^{-3} \mathrm{eV}^{2}, \sin ^{2} \theta_{23}=0.56_{-0.03}^{+0.04}, \delta_{C P}=0.0_{-0.4}^{+1.3} \pi$. 

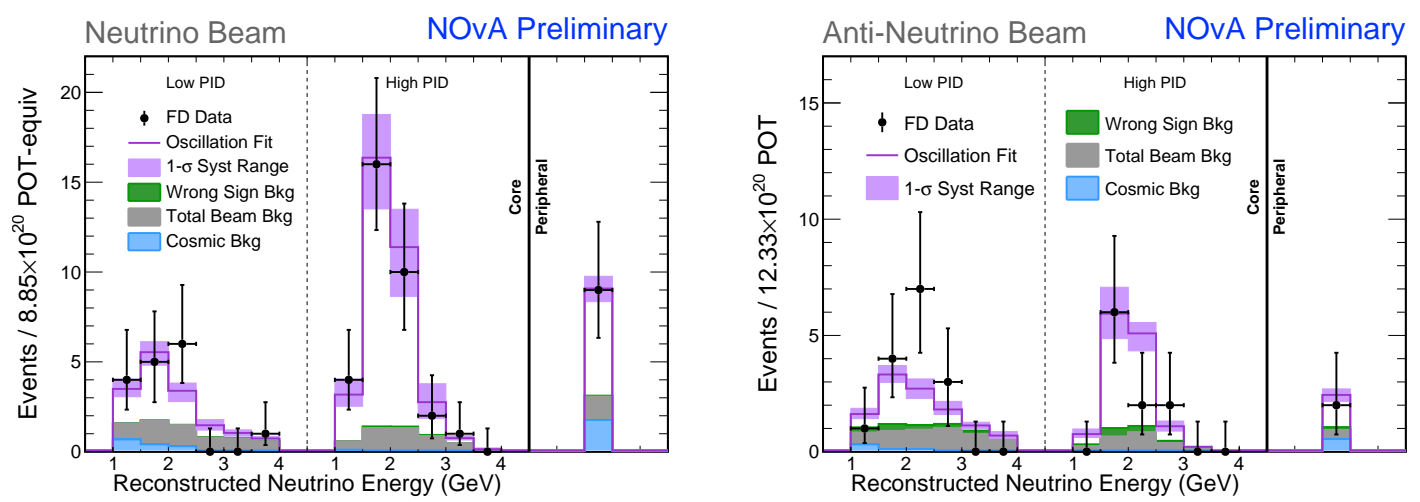

Figure 5: Measured reconstructed energy spectra of FD $v_{e} \mathrm{CC}$ (left) and $\bar{v}_{e} \mathrm{CC}$ (right) events (dots) split into two CVN PID bins and one additional Peripheral bin which collected all uncontained events included in the analysis.

This corresponds to the Normal neutrino mass hierarchy and upper octant of $\theta_{23}(>\pi / 4)$. Inverted mass hierarchy is disfavored at $1.9 \sigma$. Upper octant of $\theta_{23}$ is preferred at $1.6 \sigma$, but result is still consistent with maximal mixing at $1.2 \sigma$ (Figure 7).

The $1 \sigma, 2 \sigma, 3 \sigma$ contours in $\sin ^{2} \theta_{23}-\Delta m_{32}^{2}$ and $\sin ^{2} \theta_{23}-\delta_{C P}$ are shown in the Figure 6. A large region in Inverted hierarchy at $\delta_{C P}=\pi / 2$ was ruled out at $>4 \sigma$.

The exclusion significances at which each value of $\delta_{C P}, \Delta m_{32}^{2}$ and $\sin ^{2} \theta_{32}$ is disfavored are shown in the Figure 7. All values of $\delta_{C P}$ in the Normal hierarchy and Upper octant of $\theta_{23}$ are allowed at $1.1 \sigma$.

\section{Acknowledgments}

This work has been supported by the Russian Foundation for Basic Research under Grant no. 18-32-00935.

\section{References}

[1] M. A. Acero et al. [NOvA Collaboration], "First Measurement of Neutrino Oscillation Parameters using Neutrinos and Antineutrinos by NOvA," Phys. Rev. Lett. 123, no. 15, 151803 (2019) doi:10.1103/PhysRevLett.123.151803 [arXiv:1906.04907 [hep-ex]].

[2] D. S. Ayres et al. [NOvA Collaboration], "NOvA: Proposal to Build a 30 Kiloton Off-Axis Detector to Study $v_{\mu} \rightarrow v_{e}$ Oscillations in the NuMI Beamline," hep-ex/0503053.

[3] P. Adamson et al., "The NuMI Neutrino Beam,” Nucl. Instrum. Meth. A 806, 279 (2016) doi:10.1016/j.nima.2015.08.063 [arXiv:1507.06690 [physics.acc-ph]].

[4] A. Aurisano et al., "A Convolutional Neural Network Neutrino Event Classifier," JINST 11, no. 09, P09001 (2016) doi:10.1088/1748-0221/11/09/P09001 [arXiv:1604.01444 [hep-ex]].

[5] M. Tanabashi et al. [Particle Data Group], Phys. Rev. D 98, no. 3, 030001 (2018). doi:10.1103/PhysRevD.98.030001

[6] G. J. Feldman and R. D. Cousins, Phys. Rev. D 57, 3873 (1998) doi:10.1103/PhysRevD.57.3873 [physics/9711021 [physics.data-an]]. 

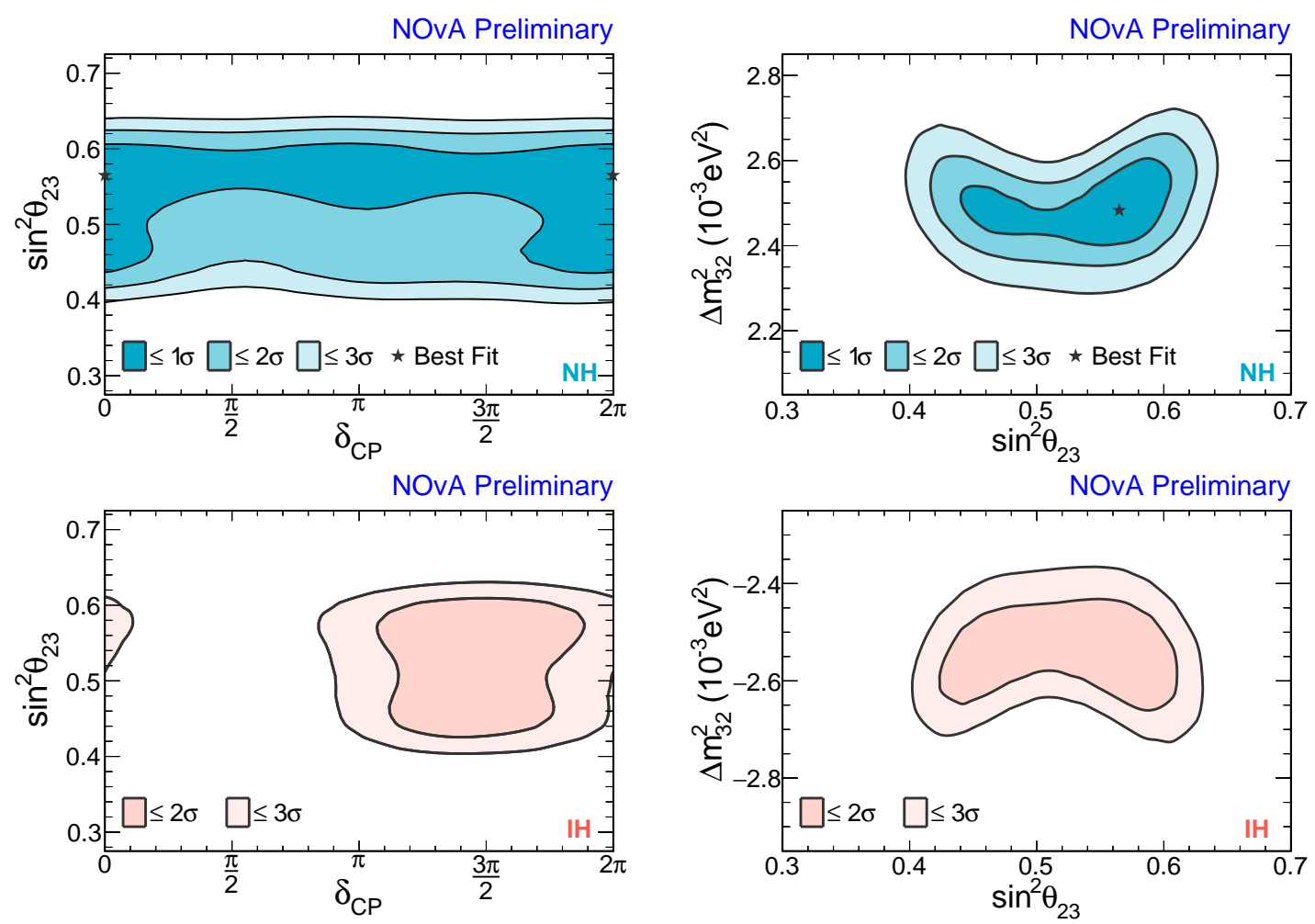

Figure 6: The $1 \sigma, 2 \sigma, 3 \sigma$ contours in $\sin ^{2} \theta_{23}-\delta_{C P}$ (left) and $\sin ^{2} \theta_{23}-\Delta m_{32}^{2}$ (right) in Normal (up row) and Inverted (bottom row) hierarchy. Feldman-Cousins corrections are applied. Best fit is shown by star marker.
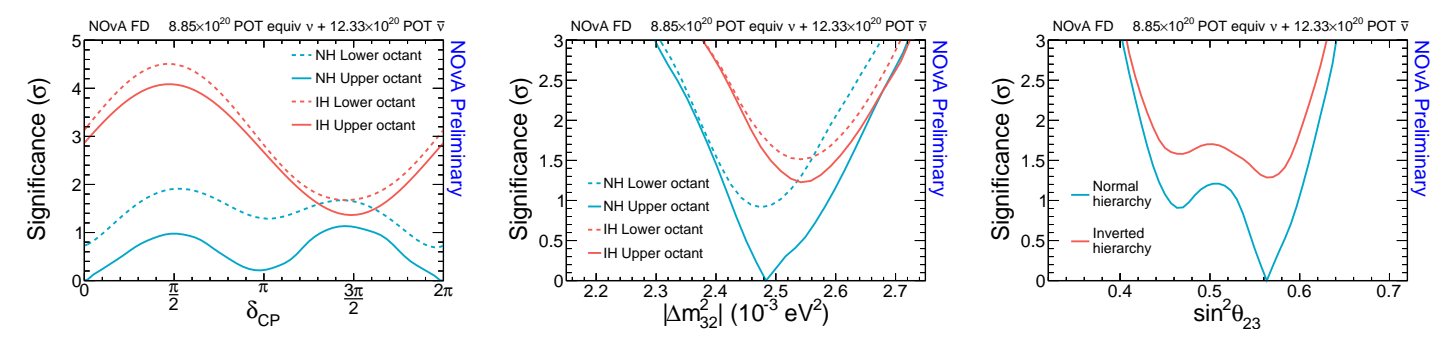

Figure 7: The exclusion significance for each value of $\delta_{C P}$ (left), $\Delta m_{32}^{2}$ (middle) and $\sin ^{2} \theta_{32}$ (right) in Normal (blue) and Inverted (orange) mass hierarchy, dashed lines denote the octant (when it's applied). 